\title{
P02-5-24 Poster session
}

\section{The effects of immunoglobulin $D$ on $T$ cell activation and differentiation in human}

\author{
Yujing Wu, Hengshi Chen, Xiaojie Dong, Wensheng Chen, Jin Dong, Xing Dai, Qiong Huang, \\ Wei Wei
}

Institute of Clinical Pharmacology, Anhui Medical University, China

OBJECTIVE To investigate the effect of immunoglobulin D ( $\operatorname{ggD}$ ) on T cell activation and imbalance of Th1/Th2, Th17/Treg cells in healthy human, and to observe whether IgD activated CD4+ T cells via Lck (Tyr394) phosphorylation. METHODS Different concentrations of IgD (final concentration of 1, 3, 10 microgram $/ \mathrm{ml}$ ) were added to stimulate the human peripheral mononuclear cells (PBMC). After 24 hours, the expression of CD69 and CD154 and the percentage of Th1, Th2, Th17 and Treg subsets were detected by flow cytometry. The expression of nuclear transcription factors T-bet, GATA-3, Foxp3 and ROR-gamma t mRNA was detected using qPCR assay. Human CD4+ T cells were purified from PBMCs with microbeads. The proliferation of $\mathrm{T}$ cells were detected by CCK- 8 . The binding affinity and expression of IgDR on $\mathrm{T}$ cells were detected by flow cytometry. The protein expression of IgDR, Lck and P-Lck were analyzed by western blot. RESULTS IgD could significantly increase the expression of CD69 and CD154, which are markers of activate T cells. IgD could simultaneously decrease the percentage of Th1 and Treg subsets and significantly increased the percentage of Th17 subset, but had no significant effect on Th2 subset. IgD significantly inhibited the expression of T-bet and Foxp3 mRNA and significantly promoted the expression of ROR-gamma t mRNA, but had no significant effect on the expression of GATA-3 mRNA. IgD could concentration-dependent bind to IgDR on CD4+ T cells. The expression of $\operatorname{IgDR}$ was increased in response to treatment with $\operatorname{IgD}$ in a time-dependent and concentration-dependent manner. Stimulating by $\operatorname{IgD}$ resulted in enhanced phosphorylation of Lck compared with that in the medium control sample. As inhibitor of PTK, Herbimycin A or A770041, which combined with IgD could significantly inhibit phosphorylation of Lck (Tyr394). The proliferation promoting effect of IgD was blocked by Herbimycin A or A770041. CONCLUSION These results demonstrate that $\operatorname{IgD}$ can activate $\mathrm{T}$ cells and induce the imbalance between Th1/Th2 and Th17/Treg subsets, which may be through promoting Lck (Tyr394) phosphorylation. 